\title{
Avaliação do Bis(4-Metilfenilditiocarbimato)Zincato(II) de Tetrabutilamônio como Acelerador no Processo de Vulcanização do Elastômero Polibutadieno
}

\author{
Leonardo F. V. dos Santos, Roberta M. Mariano, Élen B. A. V. Pacheco, Leonardo B. Canto, Leila L. Y. Visconte \\ Instituto de Macromoléculas Professora Eloisa Mano, UFRJ \\ Marcelo R. L. Oliveira, Mayura M. M. Rubinger \\ Departamento de Química, UFV
}

\begin{abstract}
Resumo: O ZNIBU, acelerador de caráter lento foi avaliado neste trabalho em composições de polibutadieno. Este acelerador foi sintetizado com o objetivo de substituir, ou reduzir, o uso de alguns aceleradores comerciais muito utilizados em composições elastoméricas mas que, por serem formadores de nitrosaminas, podem ser prejudiciais à saúde. A fim de corrigir o caráter de acelerador lento do ZNIBU, este composto foi utilizado em conjunto com o acelerador comercial CBS (N-ciclohexil-2-benzotiazol-2-sulfenamida). Foi observado que pequenas quantidades dos dois aceleradores não são efetivas na aceleração da vulcanização nas composições do tipo goma pura. No entanto a presença de negro de fumo exerce um efeito positivo sobre a velocidade. As propriedades mecânicas estudadas mostram que o ZNIBU aumenta a resistência à tração da goma pura (35\%), em comparação com a composição similar vulcanizada com CBS e, na presença do negro de fumo, este aumento é ainda mais expressivo (175\%). Quanto à resistência ao rasgamento, a vulcanização com o ZNIBU forneceu o mais baixo valor da propriedade para a goma pura, enquanto que a composição com negro de fumo apresentou o mais alto valor.
\end{abstract}

Palavras-chave: Polibutadieno, vulcanização, aceleradores, propriedades.

\section{Evaluation of [Tetrabutyl Ammonium Bis(4-Methylphenyldithiocarbimatezincate-2)] as Accelerator in Vulcanization Process of Polybutadiene Elastomer}

\begin{abstract}
ZNIBU, a slow accelerator, was evaluated in polybutadiene compositions. This accelerator was synthesized intending to replace or reduce the use of some very used commercial accelerators in rubber compositions which, due to their nitrosamine-forming potential, can be harmful to health. In order to correct for the slow accelerating character ZNIBU was used in combination with the commercial accelerator CBS (N-cyclohexyl-2-benzothiazole-2-sulfenamide). It was observed that small amounts of the accelerators are not effective in the vulcanization accelerating process of gum type compositions. Nevertheless, the presence of carbon black exerts a positive effect on the reaction rate. The investigated mechanical properties show that ZNIBU improves stress strength of the pure gum (35\%), when compared with a similar composition vulcanized with CBS and, in the presence of carbon black, the increase is even more expressive $(175 \%)$. As for the tear strength, the ZNIBU vulcanization provided the lowest value for the pure gum composition while the carbon black-filled composition presented the highest value for this property.
\end{abstract}

Keywords: Polybutadiene, vulcanization, accelerators, properties.

\section{Introdução}

A formação de ligações cruzadas em borrachas em presença apenas do enxofre como agente de vulcanização é um processo lento, mesmo a elevadas temperaturas, o que exige longos períodos de reação. Nessas condições a reticulação torna-se ineficiente, resultando em valores de resistência mecânica e propriedades de envelhecimento igualmente insatisfatórios. A duração desse procedimento somente com enxofre a temperatura de $150{ }^{\circ} \mathrm{C}$, por exemplo, é de 5 horas. A primeira tentativa de melhorar tais propriedades ocorreu com o acréscimo de óxidos que promoveu uma redução do tempo para 3 horas e, posteriormente, com o surgimento dos aceleradores, o tempo de reação passou a ser de minutos $^{[1,2]}$.

Os aceleradores permitem controlar o tempo e/ou a temperatura requerida para a vulcanização e, deste modo, não só possibilitar a cura em muito menos tempo, como também melhorar as propriedades do vulcanizado. Existem várias combinações de aceleradores usadas para aprimorar especificamente determinada propriedade. Devido à sua importância, encontram-se disponíveis no mercado vários tipos de aceleradores, que podem ser classificados de várias formas: 1) orgânicos ou inorgânicos, 2) ácidos ou básicos, 3) segundo a composição química, 4) pela velocidade de vulcanização (lenta, média, rápida e ultra-rápida) e, ainda, 5) pela estrutura química. Comercialmente, as principais famílias de aceleradores são as guanidinas, os ditiocarbamatos, os tiurans, os benzotiazóis e as sulfenamidas. Apesar de extensivamente usados pelas indústrias de artefatos de borrachas, muitos destes aceleradores tem grande potencial de geração de nitrosaminas ${ }^{[3]}$

As nitrosaminas são compostos químicos potencialmente cancerígenos de estrutura química $\mathrm{R}_{2} \mathrm{~N}-\mathrm{N}=\mathrm{O}$, produzidos a partir de nitritos e aminas. Atualmente são conhecidas cerca de 300 nitrosaminas. Estudos realizados em animais e no homem pressupõem que a exposição a quantidades suficientes destes compostos pode levar ao câncer ${ }^{[4]}$. Os tipos de câncer mais

Autor para correspondência: Roberta M. Mariano, Instituto de Macromoléculas Professora Eloisa Mano, Universidade Federal do Rio de Janeiro, 
frequentes são: da cavidade oral, pulmão, esôfago, pâncreas, fígado, nasofaringe e bexiga. As nitrosaminas estão largamente distribuídas no meio ambiente, mas é da indústria da borracha que advém as maiores concentrações. Nas borrachas, a formação de nitrosaminas ocorre durante o processo de vulcanização e surgem da reação de aminas secundárias com substâncias aceleradoras ${ }^{[5]}$.

Os sais de zinco de vários ditiocarbamatos são amplamente utilizados como aceleradores na vulcanização da borracha natural, como fardo ou em látex, devido às excelentes propriedades dos vulcanizados obtidos. Os ditiocarbamatos são um grupo de ultraaceleradores usados normalmente com outros aceleradores; são derivados do ácido ditiocarbâmico, por neutralização com uma base. Os sais de zinco são indicados para artefatos claros e sem odor, além de serem usados também como aceleradores secundários. Por serem insípidos, são recomendados também para artefatos que entrem em contato com alimentos.

O ZDMC (dimetilditiocarbamato de zinco), um dos principais aceleradores desta família, é um acelerador não manchante, usado para artefatos claros e brilhantes, com ação rápida a baixas temperaturas. O ZDEC (dietilditiocarbamato de zinco), que apresenta propriedades e aplicações semelhantes ao ZDMC, é mais utilizado em látex, uma vez que proporciona vulcanização mais rápida que o ZDMC. Já o ZBEC (dibenzilditiocarbamato de zinco), além de apresentar características semelhantes ao ZDEC, proporciona maior segurança de processamento e maior facilidade de dispersão. Todos eles possuem atividades carcinogênicas e mutagênicas devido à presença de aminas secundárias que geram nitrosaminas. Estes aceleradores são bastante utilizados, assim como os tiurans, nas plantações como pesticidas ${ }^{[6]}$.

Deve-se, então, ter muito cuidado no uso dos ditiocarbamatos por apresentarem riscos de efeitos tóxicos. Estas substâncias, sendo aminas secundárias, são produtores de $\mathrm{N}$-nitrosaminas, apresentando efeitos carcinogênicos ${ }^{[7,8]}$. O ZMDC, muito utilizado comercialmente, é classificado como um ultra-acelerador. Todavia, apresenta um mecanismo de vulcanização ainda muito obscuro. Alguns autores ${ }^{[9,10]}$ sugerem que o processo é iniciado através das reações entre o enxofre e o acelerador, resultando na formação de um complexo polissulfídico de zinco. A reatividade deste composto é explicada através do grupamento amina presente na estrutura. $\mathrm{O}$ caráter nucleofílico da substância aumenta devido à presença do grupamento amina, o que torna as ligações $\mathrm{Zn}-\mathrm{S}$ mais iônicas.

Martins e colaboradores ${ }^{[1]}$ estudaram o efeito da adição de aceleradores ditiocarmabatos em composições de EPDM (copolímero de etileno-propileno-dieno) nas propriedades reométricas e mecânicas em perfis de acabamento de tubos. As composições curaram de modo satisfatório e os vulcanizados apresentaram boa aparência e dureza aceitável, podendo absorver os impactos sem deformação.

O composto orgânico [bis(4-metilfenilditiocarbimato)zincato-2 de tetrabutilamônio], ZNIBU, é obtido a partir da reação de $\mathrm{Zn}\left(\mathrm{CH}_{3} \mathrm{COO}\right)_{2} \times 2 \mathrm{H}_{2} \mathrm{O}$ com 4-metilfenilsulfonilditiocarbimato dihidratado de potássio e brometo de tetrabutilamônio. O ZNIBU é uma substância completamente estável em condições ambientes, solúvel em clorofórmio e diclorometano, porém parcialmente solúvel em água, metanol e etanol ${ }^{[12]}$. Este composto tem sido reportado na literatura ${ }^{[13]}$ como tendo boa atividade aceleradora de vulcanização em composições de borracha natural (NR). Dessa forma, com o intuito de buscar novos compostos com potencial acelerador para outras borrachas que, ao mesmo tempo, atendessem às necessidades sociais e ambientais, o ZNIBU, cuja estrutura química é apresentada na Figura 1, foi investigado na vulcanização do polibutadieno (BR). Como o ZNIBU não apresenta em sua parte aniônica átomos de nitrogênio ligados a grupos alquila ou arila, a parte catiônica formada pelo íon tetrabutilamônio também não favorece reações de nitrosação. Portanto, esse composto não pertence à classe dos formadores de nitrosaminas secundárias, o que o torna um acelerador com grande potencial ambiental e social. $\mathrm{Na}$ literatura, a ação do ZNIBU quanto à velocidade de vulcanização foi considerada lenta ${ }^{[13]}$. Dessa forma, para sanar algumas deficiências apresentadas no uso do ZNIBU foi utilizado, neste trabalho, um sistema binário de aceleradores, composto de CBS (Nciclohexil2-benzotiazol-2-sulfenamida), um acelerador moderado, e ZNIBU, um sal composto por dois cátions tetrabutilamônio e um complexo aniônico de zinco com 4-metilfenilsulfonilditiocarbimato, em composições do elastômero polibutadieno. A avaliação do sistema binário utilizado foi feita por meio da determinação de propriedades reométricas, mecânicas e físicas.

\section{Experimental}

\section{Materiais e métodos}

A borracha utilizada neste trabalho foi o polibutadieno alto-cis, BR-40, fornecida pela Petroflex Indústria e Comércio S.A. Os demais aditivos são reagentes de grau comercial e foram utilizados como recebidos. Composições de BR, como goma pura e com carga negro de fumo (NF) (20 phr), foram preparadas em presença dos diferentes teores de aceleradores apresentados na Tabela 1. A formulação utilizada para o preparo das composições de BR foi a seguinte (em phr): polibutadieno (BR) (100); óxido de zinco (3,0); negro de fumo (0 ou 20); ácido esteárico $(2,0)$; aminox (antioxidante: produto da reação, a baixa temperatura, entre difenilamina e acetona) $(2,5)$; enxofre $(1,5)$. Os componentes foram misturados em um misturador de rolos de bancada marca Berstorff, com razão de fricção de 1:1,25 e velocidade dos rolos 24 (anterior) e 30 (posterior) rpm, durante cerca de 20 minutos (goma pura) a 30 minutos (com adição de carga), a temperatura ambiente, seguindo a ordem de adição dos ingredientes descrita na norma ASTM D3189-85.

\section{Determinação das propriedades reométricas, mecânicas e físicas}

As propriedades reométricas foram obtidas em reômetro de disco oscilatório, da marca Tecnologia Industrial, modelo TI -100 , a $150{ }^{\circ} \mathrm{C}$, operando com arco de $1^{\circ}$, segundo a norma ASTM D 2084-81, com tempo de corrida de 1 hora. A partir das curvas reométricas foram determinados os seguintes parâmetros reométricos: torque mínimo (ML), torque máximo $(\mathrm{MH})$, tempo de pré-cura (tS1) e o tempo ótimo de vulcanização (t90, tempo necessário para que $90 \%$ das ligações cruzadas sejam formadas). As propriedades mecânicas realizadas foram resistência à tração (ASTM D 412-98), resistência ao rasgamento (ASTM D 624-00), dureza shore A (ASTM D 2240-00) e deformação permanente após a compressão (DPC) (ASTM D 395-98). Os corpos de prova utilizados nos ensaios foram obtidos a partir de placas vulcanizadas em prensa hidráulica, modelo MA 098, com pressão de 15 t, a $150{ }^{\circ} \mathrm{C}$. Os ensaios de resistência à tração e resistência ao rasgamento

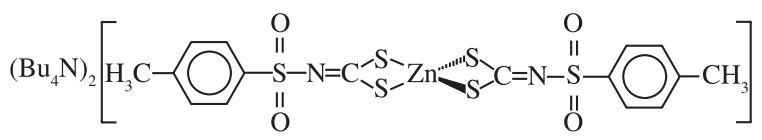

Figura 1. Estrutura química do ZNIBU.

Tabela 1. Teores de aceleradores usados nas diferentes composições.

\begin{tabular}{cccccc}
\hline Acelerador & M1 & M2 & M3 & M4 & M5 \\
\hline ZNIBU (phr) & 0,2 & 0,2 & 1,2 & 1,2 & 0,7 \\
CBS (phr) & 1,2 & 0,2 & 1,2 & 0,2 & 0,7 \\
\hline
\end{tabular}


foram realizados em máquina universal de ensaios da marca EMIC, modelo DL 3000, com célula de carga de $1 \mathrm{kN}$, de acordo com as normas correspondentes. No ensaio de DPC, a temperatura de teste foi de $70{ }^{\circ} \mathrm{C}$ e os corpos de prova foram submetidos à compressão por um período de 22 horas. O ensaio foi realizado utilizandose espaçadores metálicos e o resultado foi baseado na média dos valores obtidos.

As propriedades físicas estudadas foram a densidade relativa das composições, medida em etanol, a $23{ }^{\circ} \mathrm{C}$, segundo a norma ASTM D 297-93, a densidade de ligações cruzadas e o inchamento das composições elastoméricas, em heptano, segundo a Equação de Flory-Rehner apresentada na Equação $1^{[14,15]}$,

$$
v=\frac{-\left\{\ln (1-\mathrm{Vr})+\mathrm{Vr}+\chi \mathrm{Vr}^{2}\right\}}{\left\{\mathrm{Vo}\left(\mathrm{Vr}^{1 / 3}-\mathrm{Vr} / 2\right)\right\}}
$$

onde:

- $v$ = número de cadeias que participam do retículo $\left(\mathrm{em} \mathrm{mol} . \mathrm{cm}^{-3}\right)$;

- $\chi$ = parâmetro de interação polímero-solvente $=-0,163$;

- Vo = volume molar do solvente $\left(\mathrm{cm}^{3} \cdot \mathrm{gmol}^{-1}\right)$;

- $\mathrm{Vr}=$ fração em volume de borracha na rede inchada.

\section{Resultados e Discussão}

As misturas de aceleradores para vulcanização de borrachas são utilizadas com o objetivo de se obter vulcanizados com melhores propriedades. Neste trabalho foram feitas combinações dos aceleradores ZNIBU e CBS e os teores usados nas diferentes composições são apresentados na Tabela 1.

A Tabela 2 mostra os valores das propriedades reométricas. Pode ser observado que os dois aceleradores, quando usados isoladamente, tem características bem distintas no que se refere à capacidade aceleradora. $\mathrm{O}$ acelerador comercial CBS é classificado como um acelerador de ação moderada, enquanto que o ZNIBU apresentou comportamento de acelerador lento ${ }^{[13]}$. Em estudos realizados por Mariano e colaboradores ${ }^{[12,13]}$, a ação aceleradora do ZNIBU foi pesquisada em vulcanizados de borracha natural e foi observado que o ZNIBU apresentou ação lenta na vulcanização para composições de borracha natural, também com alto valor de $t_{90}$. Pode ser observado ainda na Tabela 2 que as composições vulcanizadas com o CBS apresentaram melhor desempenho com relação a todos os parâmetro reométricos e que a carga negro de fumo favorece a vulcanização em todos os casos, tornando-a mais rápida. A mistura $\mathrm{M} 1$ apresentou o melhor desempenho na variação de torque (parâmetro relacionado à densidade de ligações cruzadas formadas na rede do elastômero), quando comparada às demais composições desenvolvidas.

Os valores de $t_{90}$ para as misturas com sistema binário de acelerador variaram significativamente, principalmente em função da quantidade de CBS presente. Em todos os casos houve uma redução no tempo ótimo de vulcanização, em especial quando altos teores de CBS estavam associados a baixos teores de ZNIBU. Em presença da carga negro de fumo a redução foi ainda mais expressiva.

Os resultados de tempo de pré-cura das composições vulcanizadas somente com um tipo de acelerador mostraram que o CBS fornece disponibilidade maior de tempo para o processamento. Já a sua combinação com o ZNIBU levou a valores menores desse parâmetro, principalmente quando em presença de altos teores dos dois aceleradores. Isto mostra que as combinações usadas dos aceleradores não foram adequadas no que tange à melhora do tempo de pré-cura, conhecido também como tempo de $\operatorname{scorch}\left(\mathrm{t}_{\mathrm{s} 1}\right)$. A adição de negro de fumo tende a reduzir ainda mais os resultados de $t_{\mathrm{s} 1}$.

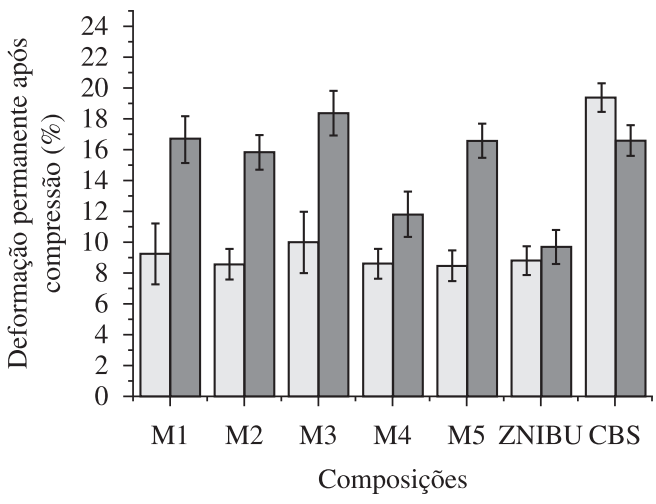

$\square$ Composição como goma pura

Composição com negro de fumo

Figura 2. Ensaio de deformação permanente após compressão (DPC) para as composições de BR como goma pura e reforçada com negro de fumo.

Tabela 2. Dados reométricos a $150{ }^{\circ} \mathrm{C}$ de composições de BR vulcanizadas com sistema binário de aceleradores ZNIBU/CBS.

\begin{tabular}{|c|c|c|c|c|c|c|}
\hline Misturas* & $\begin{array}{c}\text { Carga } \\
\text { (phr) }\end{array}$ & $\begin{array}{c}M_{L} \\
(\mathbf{d N} . \mathbf{m})\end{array}$ & $\underset{(\mathbf{d N} . \mathbf{m})}{\mathbf{M}_{\mathrm{H}}}$ & $\begin{array}{c}M_{H}-M_{L} \\
(d N . m)\end{array}$ & $\underset{\text { (minutos) }}{\mathbf{t}_{\mathrm{s}}}$ & $\stackrel{\mathrm{t}_{90}}{\text { (minutos) }}$ \\
\hline M1 $(0,2 / 1,2)$ & - & 5,6 & 33,3 & 27,7 & 4,8 & 9,6 \\
\hline \multirow{2}{*}{ M2 $(0,2 / 0,2)$} & - & 5,3 & 22,6 & 17,3 & 3,6 & 40,8 \\
\hline & 20 & 8,3 & 30,1 & 21,8 & 3,0 & 30,0 \\
\hline \multirow{2}{*}{ M3 $(1,2 / 1,2)$} & - & 5,9 & 26,6 & 20,8 & 0,6 & 24,6 \\
\hline & 20 & 7,8 & 37,0 & 29,2 & 0,6 & 10,2 \\
\hline \multirow{2}{*}{ M4 $(1,2 / 0,2)$} & - & 5,9 & 18,5 & 12,5 & 1,8 & 36,0 \\
\hline & 20 & 8,4 & 26,2 & 17,7 & 1,2 & 7,8 \\
\hline \multirow{2}{*}{ M5 $(0,7 / 0,7)$} & - & 6,1 & 26,2 & 20,1 & 1,2 & 28,8 \\
\hline & 20 & 9,0 & 38,8 & 19,8 & 0,6 & 7,2 \\
\hline \multirow{2}{*}{ CBS (0,8 phr) } & - & 6,3 & 31,4 & 20,1 & 6,0 & 20,6 \\
\hline & 20 & 7,8 & 38,1 & 30,3 & 5,4 & 14,4 \\
\hline \multirow{2}{*}{ ZNIBU (0,8 phr) } & - & 5,8 & 17,7 & 11,8 & 4,8 & 46,2 \\
\hline & 20 & 9,5 & 30,2 & 20,8 & 1,2 & 37,2 \\
\hline
\end{tabular}

*Teores na razão ZNIBU/CBS em phr; $\mathrm{M}_{\mathrm{L}}=$ torque mínimo; $\mathrm{M}_{\mathrm{H}}=$ torque máximo; $\mathrm{t}_{\mathrm{s} 1}=$ tempo de pré-cura ou $\operatorname{scorch} ; \mathrm{t}_{90}=$ tempo de $90 \%$ de cura (tempo ótimo de cura). 
O torque mínimo, $\mathrm{M}_{\mathrm{L}}$, parâmetro que está relacionado com a viscosidade e a processabilidade do material antes da vulcanização, parece não ser muito afetado pelo tipo de acelerador. Aumentos mais significativos nos valores de $\mathrm{M}_{\mathrm{L}}$ são encontrados apenas para as composições com negro de fumo, evidenciando o caráter reforçante desta carga. Quando a vulcanização está completa atinge-se o torque máximo, $\mathrm{M}_{\mathrm{H}}$, e pode-se correlacionar o grau de formação das ligações cruzadas com a diferença entre os torques máximo e mínimo, $\left(\mathrm{M}_{\mathrm{H}}-\mathrm{M}_{\mathrm{L}}\right)$. Observando-se as duas últimas linhas da Tabela 2, nota-se que o ZNIBU não é um formador de ligações cruzadas tão eficiente quanto o CBS, seja na ausência ou em presença do negro de fumo.

Observa-se também que, quando o CBS está presente em quantidades relativamente altas, como em M1, M3 e M5, a adição de ZNIBU não afeta o valor de DM. A Figura 2 mostra os resultados de deformação permanente à compressão (DPC) para as composições estudadas.

Estudos anteriores realizados com o ZNIBU ${ }^{[16]}$ mostraram que os resultados de DPC para composições de NR não foram satisfatórios. Os autores atribuíram o mau desempenho dessas composições aos tempos de vulcanização usados na preparação dos corpos de prova, considerados insuficientes para o caso do ZNIBU. Desta forma, para o desenvolvimento deste trabalho, foram preparados corpos de prova, vulcanizados com o acréscimo de 30 minutos ao tempo ótimo de vulcanização, de modo a se obter vulcanizados adequados para a preparação de corpos de prova para o ensaio de DPC.

A deformação permanente à compressão reflete a habilidade de compostos elastoméricos em reter as propriedades elásticas após a ação prolongada de uma força compressiva. O comportamento elástico da borracha de butadieno é superior ao da borracha natural. Segundo a literatura, essa característica, aliada à sua alta resistência à reversão, tem sido considerada a responsável pelos menores valores de DPC obtidos por composições de BR, quando comparadas a composições de $\mathrm{NR}^{[17]}$.

Analisando a Figura 2 foi observado que as composições vulcanizadas com o ZNIBU apresentam baixos valores de DPC, em ausência ou em presença do negro de fumo, enquanto o CBS provocou efeito inverso nas mesmas condições. Entre as demais composições, o acréscimo de negro de fumo levou ao aumento da deformação permanente, isto é, a presença da carga acarretou em uma diminuição das características elásticas dos compostos. Nesses casos, os valores mais baixos de DPC foram encontrados quando CBS foi usado em quantidades menores. Aparentemente, embora o ZNIBU não seja um acelerador tão eficiente quanto o CBS, sua presença nas composições ajuda a manter as características elásticas do polibutadieno, ao contrário do CBS.

As Figuras 3 e 4 apresentam as curvas de resistência à tração vs. alongamento para as composições de BR, em ausência e com a adição de negro de fumo, respectivamente. Os dados numéricos da propriedade são mostrados nas Tabelas 3 e 4, com os desviospadrão.

Pela observação da Figura 3, nota-se que as composições vulcanizadas com cada um dos aceleradores em separado apresentam comportamentos bem distintos com relação à resistência à tração e ao alongamento. O CBS levou aos menores valores dessas propriedades enquanto o ZNIBU promoveu uma melhora na resistência à tração e o alongamento obtido foi o maior dentre todas as composições do tipo goma pura. Quando os dois aceleradores são usados em conjunto, observa-se que maiores teores de CBS levam a valores altos de resistência à tração e baixos alongamentos na ruptura (M1, M3 e M5). Na presença de teores altos de ZNIBU e baixos de CBS, como em M4, a curva se aproxima daquela obtida para o ZNIBU puro.

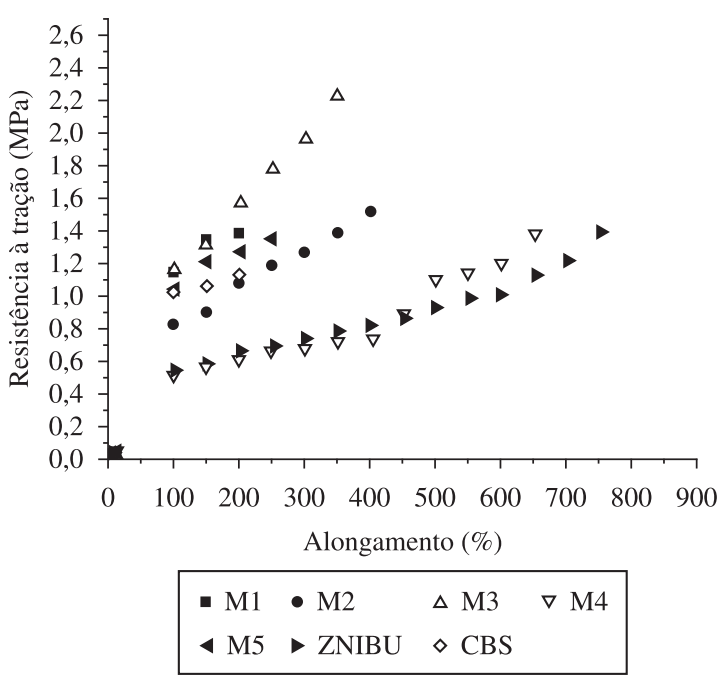

Figura 3. Resistência à tração das composições BR como goma pura.

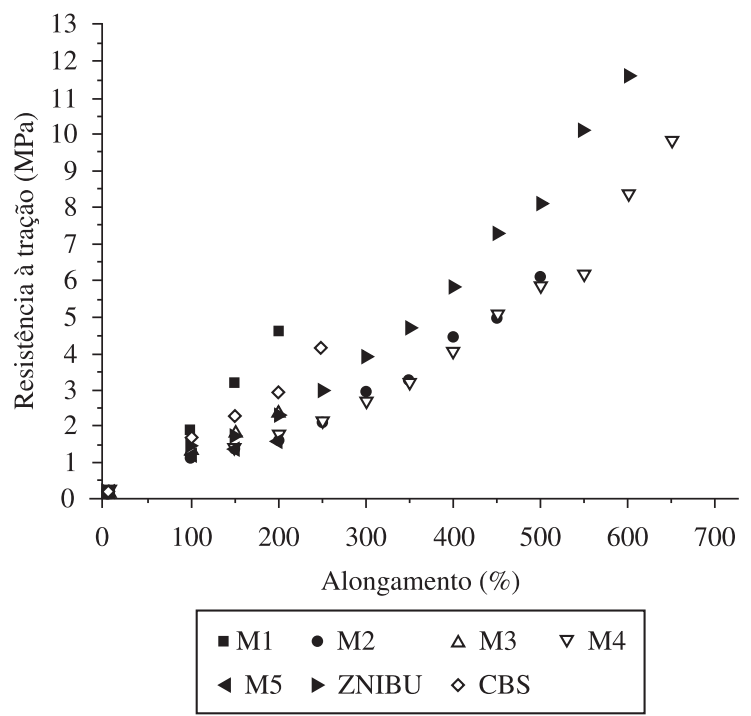

Figura 4. Resistência à tração das composições de BR reforçadas com negro de fumo.

Nas composições em que o negro de fumo é usado como carga, Figura 4, pode-se observar que a influência do CBS sobre a resistência à tração e o alongamento na ruptura é bem inferior à do ZNIBU. Os maiores valores para essas propriedades foram encontrados para a composição M4 e aquela vulcanizada com o ZNIBU apenas. Para todas as composições, à exceção de M3 e M5, houve um aumento significativo da resistência à tração com a adição do negro de fumo, o que novamente confirma o caráter reforçante da carga.

A Figura 5 apresenta os resultados de resistência ao rasgamento das composições de BR como goma pura e reforçada com negro de fumo. Comparando-se as composições do tipo goma pura, pode-se observar que o menor valor foi obtido quando o ZNIBU foi usado como único acelerador. Todas as outras gomas apresentaram valores de resistência ao rasgamento próximos ao fornecido pelo CBS. Na presença do negro de fumo a tendência se inverte e o ZNIBU fornece o maior valor para a propriedade, bem acima daquele fornecido pelo CBS, cujo valor só é superior ao dado pela composição M2. 
Tabela 3. Valores de resistência à tração em função do alongamento das composições tipo goma pura.

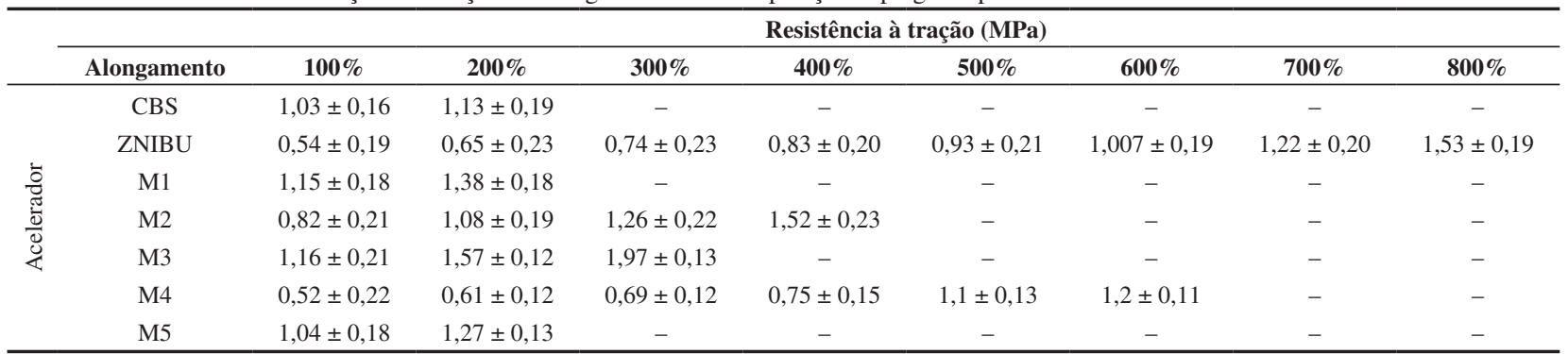

Tabela 4. Valores de resistência à tração em função do alongamento das composições reforçadas com negro de fumo.

\begin{tabular}{|c|c|c|c|c|c|c|c|}
\hline & \multirow[b]{2}{*}{ Alongamento } & \multicolumn{6}{|c|}{ Resistência à tração (MPa) } \\
\hline & & $100 \%$ & $200 \%$ & $300 \%$ & $400 \%$ & $500 \%$ & $600 \%$ \\
\hline \multirow{6}{*}{ 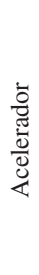 } & CBS & $1,69 \pm 0,68$ & $2,95 \pm 0,61$ & - & - & - & - \\
\hline & M1 & $1,89 \pm 0,32$ & $4,59 \pm 0,45$ & - & - & - & - \\
\hline & M2 & $1,12 \pm 0,55$ & $1,60 \pm 0,50$ & $2,92 \pm 0,55$ & $4,45 \pm 0,51$ & $6,09 \pm 0,57$ & - \\
\hline & M3 & $1,68 \pm 0,41$ & $2,33 \pm 0,40$ & - & - & - & - \\
\hline & M4 & $1,18 \pm 0,37$ & $1,77 \pm 0,40$ & $2,7 \pm 0,33$ & $4,06 \pm 0,82$ & $5,86 \pm 0,33$ & $8,36 \pm 0,39$ \\
\hline & M5 & $1,34 \pm 0,53$ & $1,59 \pm 0,62$ & - & - & - & - \\
\hline
\end{tabular}

Tabela 5. Resultados de densidade relativa das composições de BR vulcanizadas.

\begin{tabular}{cccc}
\hline Composto & $\begin{array}{c}\text { Carga } \\
(\mathbf{p h r})\end{array}$ & $\begin{array}{c}\text { Densidade } \\
\text { relativa } \\
\left(\mathbf{g . c m}^{-3}\right)\end{array}$ & $\begin{array}{c}\text { Densidade de } \\
\text { ligações cruzadas } \\
\left(\mathbf{m o l . c m}^{-3}\right) \times \mathbf{1 0}^{\mathbf{3}}\end{array}$ \\
\hline \multirow{2}{*}{ M1 } & 0 & $0,8937 \pm 0,0052$ & $1,4380 \pm 0,0040$ \\
& 20 & $0,9718 \pm 0,0096$ & $3,0802 \pm 0,0122$ \\
\hline \multirow{2}{*}{ M2 } & 0 & $0,9037 \pm 0,0124$ & $1,5532 \pm 0,0229$ \\
& 20 & $0,9624 \pm 0,0031$ & $1,7580 \pm 0,0029$ \\
\hline \multirow{2}{*}{ M3 } & 0 & $0,9197 \pm 0,0012$ & $2,5807 \pm 0,0423$ \\
& 20 & $0,9700 \pm 0,0089$ & $2,8757 \pm 0,0036$ \\
\hline \multirow{2}{*}{ M4 } & 0 & $0,8947 \pm 0,0231$ & $2,0882 \pm 0,0111$ \\
& 20 & $0,9430 \pm 0,0039$ & $2,1002 \pm 0,0356$ \\
\hline \multirow{2}{*}{ M5 } & 0 & $0,8878 \pm 0,0221$ & $2,3927 \pm 0,0237$ \\
& 20 & $0,9821 \pm 0,0075$ & $3,7405 \pm 0,0089$ \\
\hline \multirow{2}{*}{ CBS } & 0 & $0,8965 \pm 0,0321$ & $2,4488 \pm 0,0052$ \\
& 20 & $0,9852 \pm 0,0083$ & $3,2169 \pm 0,0533$ \\
\hline \multirow{2}{*}{ ZNIBU } & 0 & $0,8935 \pm 0,0122$ & $1,3851 \pm 0,0027$ \\
& 20 & $0,9616 \pm 0,0011$ & $2,7298 \pm 0,0222$ \\
\hline
\end{tabular}

Em um estudo de Wan e colaboradores ${ }^{[18]}$, composições de borracha butadiênica reforçadas com argila montmorilonita sódica in natura e organofílica foram desenvolvidas a fim de analisar o caráter reforçante da argila montmorilonita neste elastômero. Pode ser verificado que as composições de BR goma pura apresentaram valores de resistência à tração próximos a $2 \mathrm{MPa}$ enquanto que as composições com MMT-Na in natura e organofílica apresentaram valores próximos de $5 \mathrm{MPa}$ e $16 \mathrm{MPa}$, respectivamente. Embora a composição utilizada pelo autor e o método de preparo das amostras sejam diferentes dos utilizados neste trabalho, comparando superficialmente estes valores aos obtidos neste trabalho pode ser verificado que as composições de BR goma pura ou reforçadas com negro de fumo, vulzanizadas com o ZNIBU, apresentaram melhor desempenho mecânico. $\mathrm{O}$ mesmo foi verificado para propriedades de resistência ao rasgamento para as composições de BR, goma pura e reforçadas, utilizando o acelerador ZNIBU.

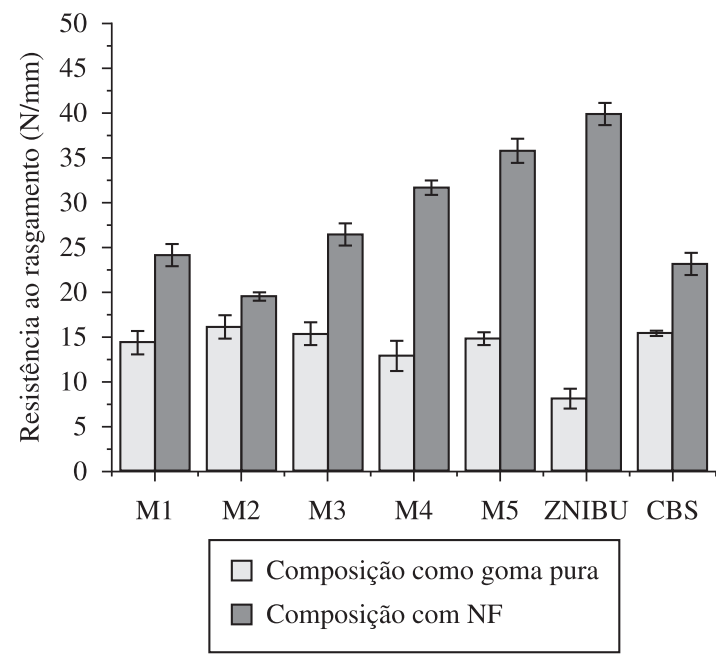

Figura 5. Resultados do ensaio de resistência ao rasgamento das composições de borracha butadiênica como goma pura e reforçado com negro de fumo.

Outros trabalhos envolvendo composições de BR, vulcanizadas empresença do aceleradorTBBS (t-butil-2-benzotiazol sulfenamida), também reportam baixos valores para a resistência à tração, o que mostra ser essa, uma característica desse elastômero ${ }^{[19,20]}$.

A Tabela 5 apresenta os resultados de ligações cruzadas das composições de BR como goma pura e reforçada com negro de fumo. Os resultados apresentados na Tabela 5 mostram que a densidade de ligações cruzadas aumenta na presença de negro de fumo e o melhor resultado é fornecido pela composição goma pura vulcanizada apenas com CBS. Foi observado que as densidades de ligações cruzadas das composições M1, M3, M5 e CBS reforçadas com negro de fumo mostraram-se semelhantes. $\mathrm{O}$ aumento do grau de ligações não favorece o desempenho em todas as propriedades mecânicas como pode ser analisado nos resultados de resistência à tração mostrados na Figura 4. As composições vulcanizadas com ZNIBU apresentaram melhores valores em relação às demais composições nos ensaios de tração. 


\section{Conclusão}

O ZNIBU foi caracterizado como um acelerador de vulcanização de ação lenta, como pode ser observado nos valores de $t_{90}$. No entanto a combinação dos dois aceleradores corrigiu esta deficiência sem prejuízo das demais propriedades, provocando o aumento do tempo de scorch e redução do tempo ótimo de vulcanização. A presença do ZNIBU nas composições ajudou a manter as características elásticas do polibutadieno, ao contrário do CBS. Os testes mecânicos mostraram que as composições de BR vulcanizadas com ZNIBU apresentaram boas propriedades, algumas delas até superiores às obtidas com $\mathrm{CBS}$, como pode ser observado nos resultados de resistência à tração das composições como goma pura e reforçadas com negro de fumo.

\section{Agradecimentos}

Os autores agradecem à Petroflex Indústria e Comércio S.A. pelo fornecimento do polibutadieno e às agências CNPq, CAPES e FAPERJ pelo apoio financeiro.

\section{Referências Bibliográficas}

1. Manuel, M. G. - "Sobre a vulcanização com enxofre”, Disponível em: http://www.rubberpedia.com/vulcanizacao/vulcanizacao-comenxofre.php. Acessado em: jun. 2009.

2. Marck, J. E.; Erman, B. \& Eirich, F. R. - "Science and technology of rubber", Academic Press, New York (1994).

3. Barlow, F. W. -"Rubber compounding: principles, materials and techniques", Marcel Dekker, INC., New York (1988).

4. Whittaker, M. H.; Gebhart, A. M.; Miller, T. C. \& Hammer, F. Toxicol. Ind. Health, 20, p.149 (2004). PMid:15941012. http://dx.doi. org/10.1191/0748233704th199oa

5. Associação Brasileira de Tecnologia da Borracha - ABTB. - Rev. Borracha Atual, 18, p.30 (1998).

6. Morton, M. - "Introduction to rubber technology", Reinhold Publishing Corporation, New York (1966).

7. Travas-Sejdic, J. - Eur. Polym. J., 32, p.1395 (1996).

8. Chasar, D. W. - Kautsch. Gummi Kunst, 42, p.31 (1989).
9. Bateman, L. - "The Chemistry and Physics of Rubber-Like Substances", Maclaren \& Sons Ltd., London (1963).

10. Nieuwenhuizen, P.J.; Reedijk, J.; Van Duin, M. \& McGill, W. J. - Rubber Chem. Technol., 70, p.368 (1997). http://dx.doi.org/10.5254/1.3538436

11. Martins, J. C.; Valim, L. P.; Rodrigues, R. D. \& Faez, R. - "Influência de parâmetros de processos nas propriedades de um composto de borracha usada em um perfil de acabamento", in: $10^{\circ}$ Encontro Latino Americano de Iniciação Científica, p.368, São José dos Campos - SP, out (2009).

12. Mariano, R. M.; Oliveira, M. R. L.; Rubinger, M. M. M. \& Visconte, L. L. Y. - Eur. Polym. J., 43, p.4706 (2007). http://dx.doi.org/10.1016/j. eurpolymj.2007.08.013

13. Mariano, R. M.; Oliveira, M. R. L.; Rubinger, M. M. M. \& Visconte, L. L. Y. - Polímeros, 18, p.297 (2008).

14. Flory, P.J. - "Principles of Polymer Chemistry", Cornell University Press, New York, 1953.

15. Mark, J. E. - Rubber Chem. Technol., 55, p.762 (1982). http://dx.doi. org/10.5254/1.3535902

16. Mariano, R. M. - "Avaliação do potencial do composto bis(4metilfenilditiocarbimato) zincato-2 de tetrabutilamônio] como acelerador de vulcanização para composições de borracha natural $(N R)$ ", Dissertação de Mestrado, Universidade Federal do Rio de Janeiro, Brasil (2007).

17. Junger, D. F. C. - "Análise térmica de misturas de borracha natural e polibutadieno: efeitos da razão elastomérica e da incorporação dos aditivos", Tese de Doutorado, Universidade Federal do Rio de Janeiro, Brasil (2007).

18. Wan, C.; Dong, W.; Zhang, Y. \& Zhang, Y. - J. Appl. Polym. Sci., 107, p.650 (2008). http://dx.doi.org/10.1002/app.27131

19. Castro, D. F.; Suarez, J. C. M.; Nunes, R. C. R. \& Visconte, L. L. Y. - J. Appl. Polym. Sci., 94, p.1575 (2004). http://dx.doi.org/10.1002/ app. 20892

20. Alcântara, A. F.; Nunes, R. C. R. \& Visconte, L. L. Y. - Polímeros, 14, p.279 (2004)

Enviado: 09/11/09

Reenviado: 18/01/11

Aceito: $24 / 01 / 11$

DOI: $10.1590 / \mathrm{S} 0104-14282011005000049$ 